\title{
The calculation of photoionization cross-section of negative hydrogen ions in the Born approximation
}

\author{
Vavrukh M. V. ${ }^{1}$, Dzikovskyi D. V. ${ }^{1}$, Stelmakh O. M. ${ }^{1}$, Solovyan V. B. ${ }^{2}$ \\ ${ }^{1}$ Ivan Franko National University of Lviv, \\ 8 Kyrylo and Methodiy Str., 79005, Lviv, Ukraine \\ ${ }^{2}$ Institute for Condensed Matter Physics of the National Academy of Sciences of Ukraine, \\ 1 Svientsitskii Str., 79011, Lviv, Ukraine
}

(Received 16 March 2020; Revised 21 March 2020; Accepted 16 April 2020)

\begin{abstract}
It is shown that the two-electron wave function of the system "hydrogen atom + photoelectron" in the Born approximation provides high accuracy of the photoionization process cross-section calculation of negative hydrogen ion. The partial cross-sections, which correspond to different "reaction ns-channels" in the scale of photoelectron energy and the scale of wavelengths are calculated. The comparisons with the results of other authors which obtained by other methods are performed.
\end{abstract}

Keywords: two-electron wave function, Born approximation, negative hydrogen ion, photoionization cross-section.

2010 MSC: $85-08$

DOI: $10.23939 / \mathrm{mmc} 2020.01 .125$

\section{Introduction}

The coefficient of continuous absorption in the stellar photospheres is formed by many processes of photons interactions with atoms of different chemical elements, their ions and electrons. In the case of stars of medium spectral types, similar to the Sun, the main mechanism is the photoionization of negative hydrogen ions, which ionization potential is $0.0555 \ldots \mathrm{Ry} \approx 0.7551 \mathrm{eV}$. Its significant contribution to the formation of the absorption coefficient is in the region $\lambda \leqslant 16.4 \cdot 10^{3} \AA$. The idea about photoionization process of $H^{-}$ions were proposed by R. Wildt [1,2], and the first approximation of cross-section calculations were performed by S. Chandrasekhar [3,4] and T. John [5]. The R. Wildt's idea was so attractive, that inspired also experimentalists. The most accurate results were obtained in the work of S. Smith and D. Burch [6], which experimentally established the spectral dependence with respect to the cross-section $\sigma(\lambda) / \sigma\left(\lambda_{0}\right)$ at $\lambda_{0}=5280 \AA$ in the region $4000-13000 \AA$. In fact, in this work was established the dependence on the wavelength of some effective hydrogen cross-section, with small contributions given by other processes. The results of theoretical works generally agreed well with the results of S. Smith and D. Burch, but could not describe the non-monotonic nature of spectral curve around its maximum, in the region $7000-8200 \AA$. In the work [7] was first shown, that the additional consideration also of the process of photoionization of excited hydrogen atoms explains the features of the experimental curve. On this basis is given the interpretation of some decrease of the continuous radiation intensity of the Sun in the region $6500-8200 \AA$, which is known from the reliable observational data, that was obtained at the end of the XX-th century $[8,9]$.

The calculations of the cross-section of photoionization process for $\mathrm{H}^{-}$ion were performed by several authors [10-14], and the deviation between the obtained results reaches $2 \%$. All works devoted to the calculation of cross-section for photon collision with $\mathrm{H}^{-}$ion, as the result of which the formed hydrogen atom is in the ground state. The accuracy of results depends on the calculation accuracy of the twoelectron wave function of the $H^{-}$ion and the wave function of the system "atom + photoelectron". Using the variational functions in this case is the general rule. The description of the system "atom + photoelectron" is similar to the problem of electron scattering on the hydrogen atom, but not identical with it. In the photoionization problem it is important to correctly describe the photoelectron state 
at close distances to the atom and their mutual influence. The best results were yielded also here with help of the variational method. In particular, in the work [10] was constructed the wave function of the system "atom + photoelectron", which has 10 variational parameters. Unfortunately, the values of these parameters are not given in the work, therefore, the role of individual inter-electron effects remained unknown.

Due to the necessity of observable features interpretation of continuous radiation of the Sun and other stars, the problem of calculation of cross-section collisions $H^{-}$ions and the hydrogen atoms with photons remains relevant, as well as the precise calculations using the alternative methods. In the present work we use the compact wave function for $\mathrm{H}^{-}$ion, calculated with basis-variational method [13]. The wave function of the system "atom + photoelectron" we obtain from the Schrödinger equation, which is reduced to the system of integro-differential equations. In the Born approximation the intermediate calculations are performed in the analytical form, and the calculation of the part of matrix elements of the electron momentum operators is reduced to the computer calculations of single integrals.

\section{General relations}

Let the outcome of the photoionization process of $\mathrm{H}^{-}$ion be a neutral hydrogen atom, in which the electron is in the state that described by the wave function $\varphi_{\sigma}(\mathbf{r})$, and the photoelectron with the wave vector $\mathbf{k}$ and the energy $\varepsilon_{k}=\hbar^{2} k^{2} / 2 m$. The process of photoionization as a quantum transition in the two-electron system in the so-called dipole approximation corresponds to the transition intensity function

$$
\frac{2}{m \hbar \omega_{\sigma, k}}\left|\left\langle\Psi_{\sigma, \mathbf{k}}\left|\left(\mathbf{e}_{q},\left[\hat{\mathbf{P}}_{1}+\hat{\mathbf{P}}_{2}\right]\right)\right| \Psi_{-}\right\rangle\right|^{2} .
$$

Here $\hat{\mathbf{P}}_{i}$ is the momentum operator of $i$-th electron, and the difference between the energies of the final and initial states of the system

$$
\hbar \omega_{\sigma, k}=\varepsilon_{k}+E_{\sigma}-E_{-}=\frac{e^{2}}{2 a_{0}}\left(k_{*}^{2}+\Delta \varepsilon+\varepsilon_{\mathrm{exc}}(\sigma)\right),
$$

where $k_{*}=k a_{0}, a_{0}$ is the Bohr radius, $\Delta \varepsilon=0.0555 \ldots$ is the ionization energy, $\varepsilon_{\text {exc }}(\sigma)=1-n_{\sigma}^{-2}$ is the excitation energy of the atom in Rydbergs, and $\mathbf{e}_{q}$ is the unit vector of electromagnetic field polarization. Furthermore, $\Psi_{-}\left(\mathbf{r}_{1}, \mathbf{r}_{2}\right)$ is the $H^{-}$ion coordinate function, which is symmetric with respect to the permutation $\mathbf{r}_{1}$ and $\mathbf{r}_{2}, \Psi_{\sigma, \mathbf{k}}\left(\mathbf{r}_{1}, \mathbf{r}_{2}\right)$ is the similar system function "atom + photoelectron". Due to the symmetry of the wave functions, the matrix elements of both electrons are the same. In the case of unpolarized light as a result of averaging over the mutual orientations of the vectors $\mathbf{e}_{q}$ and $\mathbf{P}_{\sigma}(\mathbf{k}) \equiv\left\langle\Psi_{\sigma, k}\left|\hat{\mathbf{P}}_{i}\right| \Psi_{-}\right\rangle$the function (1) can be reduced to the so-called oscillator strength

$$
f_{\sigma}(\mathbf{k})=8\left[3 m \hbar \omega_{\sigma, k}\right]^{-1}\left|\mathbf{P}_{\sigma}(\mathbf{k})\right|^{2},
$$

which determines the partial cross-section of the photoionization process for the $\sigma$-channel

$$
S_{\sigma}(\omega)=\frac{2 \pi^{2} e^{2} \hbar}{m c} \sum_{\mathbf{k}} f_{\sigma}(\mathbf{k}) \delta\left(\hbar \omega_{\sigma, k}-\hbar \omega\right),
$$

where $\hbar \omega$ is the photon energy, and the $\delta$-function provides the energy conservation law. The crosssection can also be represented in the wavelength scale, or the photoelectron energies. In this case it is convenient to use dimensionless quantities

$$
\omega_{*}=\hbar \omega\left(e^{2} / 2 a_{0}\right)^{-1}, \quad k_{*} \equiv k a_{0}, \quad \lambda_{*}=\lambda / \lambda_{0},
$$

where $\lambda_{0}=2 \pi c \hbar\left(e^{2} / 2 a_{0}\right)^{-1}=911.27 \AA$. There is the relation between these quantities as follows:

$$
k_{*}^{2}+\varepsilon_{\mathrm{exc}}(\sigma)+\Delta \varepsilon=\omega_{*}=\lambda_{*}^{-1} .
$$

Mathematical Modeling and Computing, Vol. 7, No.1, pp. 125-139 (2020) 
The full photoionization cross-section of the $H^{-}$ion is the sum of partial cross-sections that correspond to different channels

$$
S_{-}(\omega)=\sum_{\sigma} S_{\sigma}(\omega)
$$

\section{The wave function of the $\boldsymbol{H}^{-}$ion}

In the work [12], the coordinate wave function of the $H^{-}$ion is represented as the bilinear expansion by one-particle electron wave functions in neutral hydrogen atom $\varphi_{\sigma}(\mathbf{r})$

$$
\Psi_{-}\left(\mathbf{r}_{1}, \mathbf{r}_{2}\right)=\sum_{\sigma_{1}, \sigma_{2}} a_{\sigma_{1}, \sigma_{2}} \varphi_{\sigma_{1}}\left(\mathbf{r}_{1}\right) \varphi_{\sigma_{2}}\left(\mathbf{r}_{2}\right),
$$

where $a_{\sigma_{1}, \sigma_{2}}$ are the unknown coefficients, moreover $a_{\sigma_{1}, \sigma_{2}}=a_{\sigma_{2}, \sigma_{1}}$, which follows from symmetry $\Psi_{-}\left(\mathbf{r}_{1}, \mathbf{r}_{2}\right)$ of the $H^{-}$ion, which exists only in the singlet state. Substituting the expression (7) into the Schrödinger equation that describes the state of two electrons in the proton field

$$
\begin{aligned}
& \hat{H}\left(\mathbf{r}_{1}, \mathbf{r}_{2}\right) \Psi_{-}\left(\mathbf{r}_{1}, \mathbf{r}_{2}\right)=E_{-} \Psi_{-}\left(\mathbf{r}_{1}, \mathbf{r}_{2}\right), \\
& \hat{H}\left(\mathbf{r}_{1}, \mathbf{r}_{2}\right)=\hat{h}\left(\mathbf{r}_{1}\right)+\hat{h}\left(\mathbf{r}_{2}\right)+\frac{e^{2}}{r_{12}}, \quad \hat{h}\left(r_{i}\right)=-\frac{\hbar^{2} \nabla_{i}^{2}}{2 m}-\frac{e^{2}}{r_{i}},
\end{aligned}
$$

we obtained the system of homogeneous linear equations

$$
\begin{aligned}
& \sum_{\sigma_{1}^{\prime}, \sigma_{2}^{\prime}}\left\{H_{\sigma_{2}, \sigma_{1} \mid \sigma_{1}^{\prime}, \sigma_{2}^{\prime}}-E_{-} \delta_{\sigma_{1}, \sigma_{1}^{\prime}} \delta_{\sigma_{2}, \sigma_{2}^{\prime}}\right\} a_{\sigma_{1}^{\prime}, \sigma_{2}^{\prime}}=0 \\
& H_{\sigma_{2}, \sigma_{1} \mid \sigma_{1}^{\prime}, \sigma_{2}^{\prime}}=\int d \mathbf{r}_{1} \int d \mathbf{r}_{2} \varphi_{\sigma_{2}}^{*}\left(\mathbf{r}_{2}\right) \varphi_{\sigma_{1}}^{*}\left(\mathbf{r}_{1}\right) \hat{H}\left(\mathbf{r}_{1}, \mathbf{r}_{2}\right) \varphi_{\sigma_{1}^{\prime}}\left(\mathbf{r}_{1}\right) \varphi_{\sigma_{2}^{\prime}}\left(\mathbf{r}_{2}\right) .
\end{aligned}
$$

The system of equations (9) was solved by the method of perturbation theory. To this end, the superposition is used as a zero order approximation

$$
\Psi^{(0)}\left(\mathbf{r}_{1}, \mathbf{r}_{2}\right)=a_{0}^{-3} \sum_{l=1}^{k} a_{l} \psi_{l}\left(\boldsymbol{\rho}_{1}, \boldsymbol{\rho}_{2}\right)
$$

which are normalized and orthogonal functions

$$
\begin{aligned}
\psi_{1}\left(\boldsymbol{\rho}_{1}, \boldsymbol{\rho}_{2}\right) & =N_{1}(\pi \sqrt{2})^{-1}\left(\alpha_{1} \alpha_{2}\right)^{3 / 2}\left\{e^{\alpha_{1} \rho_{1}} e^{-\alpha_{2} \rho_{2}}+e^{-\alpha_{2} \rho_{1}} e^{-\alpha_{1} \rho_{2}}\right\}, \\
N_{1} & =\left\{1+\left(4 \alpha_{1} \alpha_{2}\right)^{3}\left(\alpha_{1}+\alpha_{2}\right)^{-6}\right\}^{-1 / 2} ; \\
\psi_{l}\left(\boldsymbol{\rho}_{1}, \boldsymbol{\rho}_{2}\right) & =N_{l}(\pi \sqrt{2 l-1})^{-1}\left(\gamma_{l}\right)^{2 l+1}\left(\rho_{1} \rho_{2}\right)^{l-1} P_{l-1}(t) \exp \left[-\gamma_{l}\left(\rho_{1}+\rho_{2}\right)\right]
\end{aligned}
$$

at $l \geqslant 2, P_{l-1}(t)$ is the Legendre polynomial of the order $l-1 ; t$ is the cosine of angle between the vectors $\boldsymbol{\rho}_{1}$ and $\boldsymbol{\rho}_{2}, \boldsymbol{\rho}_{i}=\mathbf{r}_{i} / a_{0} ; N_{2}=1 ; N_{3}=2 / 9 ; N_{4}=1 / 45, \ldots$; the parameters $\alpha_{1}, \alpha_{2}$ and $\gamma_{l}$ are the variational ones. The coefficients $a_{l}$ are determined from the secular problem, and the parameters $\alpha_{1}, \alpha_{2}, \gamma_{l}$ from the minimum energy condition of $H^{-}$ion. In the case $k=4$ the ion energy in such approximation equals $E_{0}=-1.05239 \mathrm{Ry}$,

$$
\begin{array}{lllll}
a_{1}=0.9939285, & a_{2}=-0.1086079, & a_{3}=-0.0176175, & a_{4}=-0.005792 ; & \\
\alpha_{1}=1.03524, & \alpha_{2}=0.326516, & \gamma_{2}=1.00138, & \gamma_{3}=1.53401, & \gamma_{4}=2.09053 .
\end{array}
$$

The expansion coefficients $\Psi^{(0)}\left(\mathbf{r}_{1}, \mathbf{r}_{2}\right)$ for the basis functions $\varphi_{\sigma}(\mathbf{r})$

$$
A_{\sigma_{1}, \sigma_{2}}=\iint d \mathbf{r}_{1} d \mathbf{r}_{2} \Psi^{(0)}\left(\mathbf{r}_{1}, \mathbf{r}_{2}\right) \varphi_{\sigma_{1}}^{*}\left(\mathbf{r}_{1}\right) \varphi_{\sigma_{2}}^{*}\left(\mathbf{r}_{2}\right)
$$

were used as a zero approximation to find the solutions of the system (9). Using the substitution

$$
a_{\sigma_{1}, \sigma_{2}}=A_{\sigma_{1}, \sigma_{2}}+\alpha_{\sigma_{1}, \sigma_{2}}
$$

Mathematical Modeling and Computing, Vol. 7, No. 1, pp. 125-139 (2020) 
the system (9) is reduced to the system of linear inhomogeneous equations for the unknowns $\alpha_{\sigma_{1}, \sigma_{2}}$

$$
\begin{aligned}
& \sum_{\sigma_{1}^{\prime}, \sigma_{2}^{\prime}} \alpha_{\sigma_{1}^{\prime}, \sigma_{2}^{\prime}} H_{\sigma_{2}, \sigma_{1} \mid \sigma_{1}^{\prime}, \sigma_{2}^{\prime}}-E_{-} \alpha_{\sigma_{1}, \sigma_{2}}=E_{-} A_{\sigma_{1}, \sigma_{2}}-H_{\sigma_{1}, \sigma_{2}}, \\
& H_{\sigma_{1}, \sigma_{2}}=\iint d \mathbf{r}_{1} d \mathbf{r}_{2} \varphi_{\sigma_{2}}^{*}\left(\mathbf{r}_{2}\right) \varphi_{\sigma_{1}}^{*}\left(\mathbf{r}_{1}\right) \hat{H}\left(\mathbf{r}_{1}, \mathbf{r}_{2}\right) \Psi^{(0)}\left(\mathbf{r}_{1}, \mathbf{r}_{2}\right),
\end{aligned}
$$

and

$$
E_{-}=E_{0}+\sum_{\sigma_{1}, \sigma_{2}} \alpha_{\sigma_{1}, \sigma_{2}}\left\{H_{\sigma_{1}, \sigma_{2}}^{*}-E_{-} A_{\sigma_{1}, \sigma_{2}}^{*}\right\}
$$

The coefficients $\alpha_{\sigma_{1}, \sigma_{2}}$ were found in the first approximation of the perturbation theory,

$$
\begin{aligned}
\alpha_{\sigma_{1}, \sigma_{2}}^{(1)} & =\left\{E_{0} A_{\sigma_{1}, \sigma_{2}}-H_{\sigma_{1}, \sigma_{2}}\right\}\left\{\tilde{H}_{\sigma_{1}, \sigma_{2}}-E_{0}\right\}^{-1}, \\
\tilde{H}_{\sigma_{1}, \sigma_{2}} & =H_{\sigma_{2}, \sigma_{1} \mid \sigma_{1}, \sigma_{2}}-\left(1-\delta_{\sigma_{1}, \sigma_{2}}\right) H_{\sigma_{2}, \sigma_{1} \mid \sigma_{2}, \sigma_{1}},
\end{aligned}
$$

as a result of which the energy and the wave function of $H^{-}$ion are following

$$
\begin{aligned}
& E_{-}^{(1)}=E_{0}+\sum_{\sigma_{1}, \sigma_{2}} \Delta E_{\sigma_{1}, \sigma_{2}} ; \quad \Delta E_{\sigma_{1}, \sigma_{2}}=\alpha_{\sigma_{1}, \sigma_{2}}^{(1)}\left\{H_{\sigma_{1}, \sigma_{2}}^{*}-E_{0} A_{\sigma_{1}, \sigma_{2}}^{*}\right\} \\
& \Psi_{-}^{(1)}\left(\mathbf{r}_{1}, \mathbf{r}_{2}\right)=\Psi^{(0)}\left(\mathbf{r}_{1}, \mathbf{r}_{2}\right)+\sum_{\sigma_{1}, \sigma_{2}} \alpha_{\sigma_{1}, \sigma_{2}}^{(1)} \varphi_{\sigma_{1}}\left(\mathbf{r}_{1}\right) \varphi_{\sigma_{2}}\left(\mathbf{r}_{2}\right) .
\end{aligned}
$$

The calculations were performed using the hydrogen functions $\varphi_{1 s}(\mathbf{r}), \varphi_{2 s}(\mathbf{r}), \varphi_{3 s}(\mathbf{r}), \varphi_{2 p, m}(\mathbf{r})$, where $m=0, \pm 1$. In such approximation the $H^{-}$ion energy becomes $E_{-}=1.055472 \ldots$ Ry, moreover the corrections $\Delta E_{\sigma_{1} \sigma_{2}}<0$.

It should be noted that the 5-parameter function $\Psi^{(0)}\left(\mathbf{r}_{1}, \mathbf{r}_{2}\right)$ gives the same value of $H^{-}$ion energy as the R. Tweed's function with 31 or 41 parameters [15], or 20-parametric J. Hart and G. Herzberg function [16]. The $H^{-}$ion energy which was obtained in the work [12] differs from the result of the work [17], in which the variational function with 444 parameters was used, only on $2.7 \cdot 10^{-5} \mathrm{Ry}$.

\section{The wave function of the system "atom + photoelectron"}

To describe the systems with localized and delocalized electron states, it is natural to use the reference system approach [18]. In this approach the wave function of the system "atom + photoelectron" can be written in the form of expansion

$$
\Psi_{\sigma, \mathbf{k}}\left(\mathbf{r}_{1}, \mathbf{r}_{2}\right)=\frac{\mathcal{N}}{\sqrt{2}} \sum_{\sigma_{1}}\left\{\varphi_{\sigma_{1}}\left(\mathbf{r}_{1}\right) F_{\sigma_{1}}\left(\mathbf{k} \mid \mathbf{r}_{2}\right)+\varphi_{\sigma_{1}}\left(\mathbf{r}_{2}\right) F_{\sigma_{1}}\left(\mathbf{k} \mid \mathbf{r}_{1}\right)\right\}
$$

$\mathcal{N}$ is the normalization constant. In order to make this function satisfy the $\sigma$-channel of reaction, when in the result of ionization formed neutral atom with the electron in the state $\varphi_{\sigma}(r)$ and photoelectron with the wave vector $\mathbf{k}$ and the energy $\varepsilon_{k}=\hbar^{2} k^{2} / 2 m$, the next conditions must be fulfilled

$$
\begin{aligned}
& \lim _{r \rightarrow \infty} F_{\sigma}(\mathbf{k} \mid \mathbf{r})=\varphi_{\mathbf{k}}^{(0)}(\mathbf{r})=V^{-1 / 2} \exp [i(\mathbf{k}, \mathbf{r})], \\
& \lim _{r \rightarrow \infty} F_{\sigma_{1}}(\mathbf{k} \mid \mathbf{r})=0 \quad \text { at } \quad \sigma_{1} \neq \sigma
\end{aligned}
$$

Therefore, the main term in the expression (19) is the term $\sigma_{1}=\sigma$, which corresponds to the model of "hard" atoms, and terms $\sigma_{1} \neq \sigma$ play the role of corrections that take into account the atom polarization by photoelectron. The wave function (19) and the function $\Psi_{-}\left(\mathbf{r}_{1}, \mathbf{r}_{2}\right)$ are the eigenfunctions of the same Hamiltonian, which correspond to different energy values, therefore they must be orthogonal to 
each other. The sufficient condition for this is the orthogonality of unknowns $F_{\sigma_{1}}(\mathbf{k} \mid \mathbf{r})$ to the atomic wave functions

$$
\int F_{\sigma_{1}}(\mathbf{k} \mid \mathbf{r}) \varphi_{\sigma_{2}}^{*}(\mathbf{r}) d \mathbf{r}=0
$$

At the approximate calculations this condition can be used in the optimization procedure for finding correct approximations for the functions $F_{\sigma_{1}}(\mathbf{k} \mid \mathbf{r})$.

For the purpose of clearness, we consider the first case of $1 s$-channel of reaction, when the ionization product is the hydrogen atom in the ground state $(\sigma=1 s)$. This channel is most important, because it corresponds to the cross-section, which is non-zero in the range $0<\lambda<16.4 \cdot 10^{3} \AA$ and only this channel was considered in the works of our predecessors. The another channels of reactions, which we will consider later, correspond to the cross-section, which are non-zero in the range of deep ultraviolet $(\lambda<1241.5 \AA)$.

Substituting the expression (19) in the Schrödinger equation and taking into account the expression (21), we obtain the system of coupled integro-differential equations for the functions

$$
\begin{aligned}
\left\{\frac{\hbar^{2} \nabla^{2}}{2 m}-\varepsilon_{k}+E_{\sigma_{1}}-E_{1 s}\right. & \left.+\nu_{\sigma_{1}}(r)\right\} F_{\sigma_{1}}(\mathbf{r}) \\
& +\varphi_{\sigma_{1}}(\mathbf{r}) \int \varphi_{\sigma_{1}}^{*}\left(\mathbf{r}_{2}\right) \frac{e^{2}}{\left|\mathbf{r}-\mathbf{r}_{2}\right|} F_{\sigma_{1}}\left(\mathbf{r}_{2}\right) d \mathbf{r}_{2}=-\sum_{\sigma^{\prime}\left(\neq \sigma_{1}\right)} \hat{M}_{\sigma_{1}}(\mathbf{r}) F_{\sigma^{\prime}}(\mathbf{r}) .
\end{aligned}
$$

Here the following notation is used

$$
\begin{aligned}
\hat{M}_{\sigma^{\prime}}(\mathbf{r}) F_{\sigma^{\prime}}(\mathbf{r}) & =\omega_{\sigma_{1} \sigma^{\prime}}(\mathbf{r}) F_{\sigma^{\prime}}(\mathbf{r})+\varphi_{\sigma^{\prime}}(\mathbf{r}) \int \varphi_{\sigma_{1}}^{*}\left(\mathbf{r}_{2}\right) \frac{e^{2}}{\left|\mathbf{r}-\mathbf{r}_{2}\right|} F_{\sigma^{\prime}}\left(\mathbf{r}_{2}\right) d \mathbf{r}_{2}, \\
\omega_{\sigma_{1} \sigma^{\prime}}(\mathbf{r}) & =\int \varphi_{\sigma_{1}}^{*}\left(\mathbf{r}_{2}\right) \frac{e^{2}}{\left|\mathbf{r}-\mathbf{r}_{2}\right|} \varphi_{\sigma^{\prime}}\left(\mathbf{r}_{2}\right) d \mathbf{r}_{2} \\
\nu_{\sigma_{1}}(\mathbf{r}) & =-\frac{e^{2}}{r}+\omega_{\sigma_{1}, \sigma^{\prime}}(\mathbf{r}) .
\end{aligned}
$$

So, the left side of the equation (22) takes into account electrostatic and exchange effects of "hard" model of atom, and the right side describes the analogous influence effects of different states $\left(\sigma^{\prime} \neq \sigma_{1}\right)$ on the formation $F_{\sigma_{1}}(\mathbf{r})$.

In order to find approximate solutions of the system (22) we transform it to the integral form. Let us introduce a set of Green's functions $G_{\sigma_{1}}^{(0)}\left(\mathbf{r}, \mathbf{r}^{\prime}\right)$, which satisfy the equation

$$
\left\{\frac{\hbar^{2} \nabla^{2}}{2 m}-\varepsilon_{k}+E_{\sigma_{1}}-E_{1 s}\right\} G_{\sigma_{1}}^{(0)}\left(\mathbf{r}, \mathbf{r}^{\prime}\right)=\delta\left(\mathbf{r}-\mathbf{r}^{\prime}\right) .
$$

Using the solutions of equation (24)

$$
G_{\sigma_{1}}^{(0)}\left(\mathbf{r}, \mathbf{r}^{\prime}\right)=V^{-1} \sum_{\mathbf{q}}\left\{\varepsilon_{q}-\varepsilon_{k}+E_{\sigma_{1}}-E_{1 s}\right\}^{-1} \exp \left[i\left(\mathbf{q}, \mathbf{r}-\mathbf{r}^{\prime}\right)\right]
$$

we write the system of equations (22) in the integral form:

$$
\begin{aligned}
F_{\sigma_{1}}(\mathbf{r}) & =F_{\sigma_{1}}^{(0)}(\mathbf{r})-\int G_{\sigma_{1}}^{(0)}\left(\mathbf{r}, \mathbf{r}^{\prime}\right) \Phi_{\sigma_{1}}\left(\mathbf{r}^{\prime}\right) d \mathbf{r}^{\prime}, \\
\Phi_{\sigma_{1}}\left(\mathbf{r}^{\prime}\right) & =\nu_{\sigma_{1}}\left(\mathbf{r}^{\prime}\right) F_{\sigma_{1}}\left(\mathbf{r}^{\prime}\right)+\varphi_{\sigma_{1}}\left(\mathbf{r}^{\prime}\right) \int \varphi_{\sigma_{1}}^{*}\left(\mathbf{r}_{2}\right) \frac{e^{2}}{\left|\mathbf{r}^{\prime}-\mathbf{r}_{2}\right|} F_{\sigma_{1}}\left(\mathbf{r}_{2}\right) d \mathbf{r}_{2}+\sum_{\sigma^{\prime}\left(\neq \sigma_{1}\right)} \hat{M}_{\sigma^{\prime}}\left(\mathbf{r}^{\prime}\right) F_{\sigma^{\prime}}\left(\mathbf{r}^{\prime}\right) .
\end{aligned}
$$

The functions $F_{\sigma_{1}}^{(0)}(\mathbf{r})$ are the solutions of equations

$$
\left\{-\frac{\hbar^{2} \nabla^{2}}{2 m}-\varepsilon_{k}+E_{\sigma_{1}}-E_{1 s}\right\} F_{\sigma_{1}}^{(0)}(\mathbf{r})=0 .
$$

Mathematical Modeling and Computing, Vol. 7, No. 1, pp. 125-139 (2020) 
In accordance with the asymptotics (20) the function $F_{1 s}(\mathbf{r})$ is a plane wave, and the solutions (27) at $\sigma_{1} \neq 1 s$ must be the localized functions with condition $\varepsilon_{k}<E_{\sigma_{1}}-E_{1 s}$. In the case $\varepsilon_{k}>E_{\sigma_{1}}-E_{1 s}$ we assume $F_{\sigma_{1}}^{(0)}(\mathbf{r})=0$. Let us introduce the projection operator

$$
\hat{P}=1-\sum_{\sigma}\left|\varphi_{\sigma}\right\rangle\left\langle\varphi_{\sigma}\right|
$$

Acting on the plane wave $\varphi_{\mathbf{k}}^{(0)}(r)$, this operator forms a so-called orthogonalized plane wave,

$$
\hat{P} \varphi_{\mathbf{k}}^{(0)}(r)=\varphi_{\mathbf{k}}^{(0)}(r)-\sum_{\sigma}\left\langle\varphi_{\sigma} \mid \varphi_{\mathbf{k}}^{(0)}\right\rangle \varphi_{\sigma}(\mathbf{r}) \equiv \varphi_{\mathbf{k}}(\mathbf{r})
$$

which is orthogonalized to the atomic functions $\varphi_{\sigma}(\mathbf{r})$ by definition. According to the condition (21) $\hat{P} F_{\sigma_{1}}(\mathbf{r})=F_{\sigma_{1}}(\mathbf{r})$. In those cases when $F_{\sigma_{1}}^{(0)}(\mathbf{r})$ is the localized function, it can be represented in the form of expansion for the functions $\varphi_{\sigma}(\mathbf{r})$, but because of that $\hat{P} F_{\sigma_{1}}^{(0)}(\mathbf{r})=0$ at $\sigma_{1} \neq 1 s$.

Acting on the left and right sides of the equations (26) with the operator $\hat{P}$, we transform them to such form:

$$
F_{\sigma_{1}}(\mathbf{r})=\varphi_{\mathbf{k}}(\mathbf{r}) \delta_{\sigma_{1}, 1 s}-\int G_{\sigma_{1}}\left(\mathbf{r}, \mathbf{r}^{\prime}\right) \Phi_{\sigma_{1}}\left(\mathbf{r}^{\prime}\right) d \mathbf{r}^{\prime},
$$

where $\delta_{\sigma_{1}, 1 s}$ is the Kronecker symbol,

$$
G_{\sigma_{1}}\left(\mathbf{r}, \mathbf{r}^{\prime}\right)=V^{-1 / 2} \sum_{\mathbf{q}}\left\{\varepsilon_{q}-\varepsilon_{k}+E_{\sigma_{1}}-E_{1 s}\right\}^{-1} \varphi_{\mathbf{q}}(\mathbf{r}) \exp \left[-i\left(\mathbf{q}, \mathbf{r}^{\prime}\right)\right]
$$

To solve the equations (30) we apply the iteration method. In the first Born approximation

$$
\begin{aligned}
F_{1 s}^{(1)}(\mathbf{r}) & =\varphi_{\mathbf{k}}(\mathbf{r})-\int G_{1 s}\left(\mathbf{r}, \mathbf{r}^{\prime}\right)\left\{\nu_{1 s}\left(\mathbf{r}^{\prime}\right) \varphi_{\mathbf{k}}\left(\mathbf{r}^{\prime}\right)+\varphi_{1 s}\left(\mathbf{r}^{\prime}\right) \int d \mathbf{r}_{2} \varphi_{1 s}\left(\mathbf{r}_{2}\right) \frac{e^{2}}{\left|\mathbf{r}^{\prime}-\mathbf{r}_{2}\right|} \varphi_{\mathbf{k}}\left(\mathbf{r}_{2}\right)\right\} d \mathbf{r}^{\prime} \\
& =\varphi_{\mathbf{k}}(\mathbf{r})+\chi_{1 s}(\mathbf{k} \mid \mathbf{r}) \\
\nu_{1 s}(\mathbf{r}) & =-\frac{e^{2}}{r}\left(1+r / a_{0}\right) e^{-2 r / a_{0}},
\end{aligned}
$$

and at $\sigma_{1} \neq 1 s$

$$
\begin{aligned}
F_{\sigma_{1}}^{(1)}(\mathbf{r}) & =-\int G_{\sigma_{1}}\left(\mathbf{r}, \mathbf{r}^{\prime}\right)\left\{\omega_{\sigma_{1}, 1 s}\left(\mathbf{r}^{\prime}\right) \varphi_{\mathbf{k}}\left(\mathbf{r}^{\prime}\right)+\varphi_{1 s}\left(\mathbf{r}^{\prime}\right) \int d \mathbf{r}_{2} \varphi_{\sigma_{1}}^{*}\left(\mathbf{r}_{2}\right) \frac{e^{2}}{\left|\mathbf{r}^{\prime}-\mathbf{r}_{2}\right|} \varphi_{\mathbf{k}}\left(\mathbf{r}_{2}\right)\right\} d \mathbf{r}^{\prime} \\
& =\chi_{\sigma_{1}}(\mathbf{k} \mid \mathbf{r}) .
\end{aligned}
$$

The application of such approximation is justified by the fact that the corrections $\chi_{1 s}(\mathbf{k} \mid \mathbf{r})$ and $\chi_{\sigma_{1}}(\mathbf{k} \mid \mathbf{r})$ have built on orthogonalized plane waves, and electrostatic and exchange potentials are weak.

Integration over the variables $\mathbf{r}^{\prime}$ and $\mathbf{r}_{2}$ in the expressions (32), (33) is easy to perform, using the expansions of potentials and plane waves $\varphi_{\mathbf{k}}^{(0)}\left(\mathbf{r}_{2}\right), \varphi_{\mathbf{q}}^{(0)}\left(\mathbf{r}^{\prime}\right)$ by the spherical harmonics:

$$
\begin{aligned}
& \exp \left[i\left(\mathbf{k}, \mathbf{r}^{\prime}\right)\right]=\sum_{l=0}^{\infty}(2 l+1)(i)^{l} P_{l}\left(\widehat{\mathbf{k}_{*}, \boldsymbol{\rho}}\right) j_{l}\left(k_{*} \rho\right), \quad k_{*}=k a_{0} \\
& \frac{e^{2}}{\left|\mathbf{r}_{1}-\mathbf{r}_{2}\right|}=\frac{e^{2}}{a_{0}} \sum_{l=0}^{\infty} P_{l}\left(\widehat{\boldsymbol{\rho}_{1}, \boldsymbol{\rho}_{2}}\right) a_{l}\left(\rho_{1}, \rho_{2}\right), \quad P_{l}\left(\widehat{\boldsymbol{\rho}_{1}, \boldsymbol{\rho}_{2}}\right)=\sum_{m=-l}^{l} \frac{4 \pi}{2 l+1} Y_{l, m}^{*}\left(\boldsymbol{\rho}_{1}\right) Y_{l, m}\left(\boldsymbol{\rho}_{2}\right), \\
& P_{l}\left(\widehat{\mathbf{k}_{*}, \boldsymbol{\rho}}\right) \equiv \sum_{m=-l}^{l} \frac{4 \pi}{2 l+1} Y_{l m}^{*}\left(\mathbf{k}_{*}\right) Y_{l m}(\boldsymbol{\rho}),
\end{aligned}
$$

where $a_{l}\left(\rho_{1}, \rho_{2}\right)=\rho_{1}^{-l} \rho_{2}^{l+1}$ at $\rho_{1}>\rho_{2}, a_{l}\left(\rho_{1}, \rho_{2}\right)=\rho_{2}^{-l} \rho_{1}^{l+1}$ at $\rho_{2}>\rho_{1} ; P_{l}\left(\widehat{\mathbf{a}_{1}, \mathbf{a}_{2}}\right)$ is the Legendre polynomial of $l$-th order from cosine of angle between vectors $\mathbf{a}_{1}$ and $\mathbf{a}_{2} ; Y_{l m}(\boldsymbol{\rho})$ is the spherical function, which depend on the angular variables of the vector $\boldsymbol{\rho}, Y_{l m}\left(\mathbf{k}_{*}\right)$ is the same function of 
angular variables of the vector $\mathbf{k}_{*} ; j_{l}(x)$ is the spherical Bessel function of $l$-th order of first kind [19]. It allows us to rewrite $\chi_{1 s}(\mathbf{k} \mid \mathbf{r})$ in such form:

$$
\chi_{1 s}(\mathbf{k} \mid \mathbf{r})=-\frac{4 \pi e^{2} a_{0}^{2}}{V} \sum_{\mathbf{q}} \frac{\varphi_{\mathbf{q}}(\mathbf{r})}{\varepsilon_{q}-\varepsilon_{k}} \sum_{l=0}^{\infty}(2 l+1) P_{l}(\hat{\mathbf{k}, \mathbf{q}}) \Xi_{l}^{1 s}\left(k_{*}, q_{*}\right) .
$$

Herewith $P_{l}(\widehat{\mathbf{k}, \mathbf{q}})$ is the Legendre polynomial from cosine of angle between vectors $\mathbf{k}$ and $\mathbf{q}$;

$$
\Xi_{l}^{1 s}\left(k_{*}, q_{*}\right)=\left\{8 a_{l}^{1 s}\left(k_{*}, q_{*}\right)+\nu_{l}^{1 s}\left(k_{*}, q_{*}\right)\right\}-\sum_{n=l+1}^{\infty} \tilde{q}_{n, l}^{*}\left(k_{*}\right)\left\{8 b_{n, l}^{1 s}\left(q_{*}\right)+\nu_{n, l}^{1 s}\left(q_{*}\right)\right\}
$$

is the scalar function of dimensionless variables $k_{*}$ and $q_{*}$. Here we used such notation $\left(q_{*}=q a_{0}\right)$ :

$$
\begin{aligned}
a_{l}^{1 s}\left(k_{*}, q_{*}\right) & =[2(2 l+1)]^{-1} \iint_{0}^{\infty} d \rho_{1} d \rho_{2}\left(\rho_{1} \rho_{2}\right)^{2} e^{-\rho_{1}} e^{-\rho_{2}} a_{l}\left(\rho_{1}, \rho_{2}\right) j_{l}\left(k_{*} \rho_{1}\right) j_{l}\left(q_{*} \rho_{2}\right) ; \\
\nu_{l}^{1 s}\left(k_{*}, q_{*}\right) & =-\int_{0}^{\infty} d \rho \rho(1+\rho) e^{-2 \rho} j_{l}\left(k_{*} \rho\right) j_{l}\left(q_{*} \rho\right) ; \\
b_{n, l}^{1 s}\left(q_{*}\right) & =[2(2 l+1)]^{-1} \iint_{0}^{\infty} d \rho_{1} d \rho_{2}\left(\rho_{1} \rho_{2}\right)^{2} a_{l}\left(\rho_{1}, \rho_{2}\right) e^{-\left(\rho_{1}+\rho_{2}\right)} R_{n, l}\left(\rho_{2}\right) j_{l}\left(q_{*} \rho_{1}\right) ; \\
\nu_{n, l}^{1 s}\left(q_{*}\right) & =\int_{0}^{\infty} d \rho \rho_{2} R_{n, l}(\rho) j_{l}\left(q_{*} \rho\right) \nu_{1 s}(\rho), \quad \nu_{1 s}(\rho)=-\frac{1}{9}(1+\rho) e^{-2 \rho} ;
\end{aligned}
$$

where $R_{n, l}(\rho)$ is the radial factor of atomic function $\varphi_{\sigma}(\mathbf{r})$ at $\sigma=(n, l, m)$;

$$
\tilde{q}_{n, l}\left(k_{*}\right)=(-i)^{l} \int_{0}^{\infty} d \rho \rho^{2} R_{n, l}(\rho) j_{l}\left(k_{*} \rho\right) .
$$

The factor $\left\{8 a_{l}^{1 s}\left(k_{*}, q_{*}\right)-\nu_{1}^{1 s}\left(k_{*}, q_{*}\right)\right\}$ in the formula (36) corresponds to the plane-wave approximation, and all others - account for orthogonalization components. The convergence of the expansion relative to the orbital wave number $l$ in the formula (36) in the case of low-energy photons $\left(\right.$ at $\left.k_{*} \ll 1\right)$ is provided by the asymptotics of the coefficient functions

$$
a_{l}^{1 s}\left(k_{*}, q_{*}\right) \sim\left(k_{*}\right)^{l}, \quad \nu_{l}^{1 s}\left(k_{*}, q_{*}\right) \sim\left(k_{*}\right)^{l}, \quad \tilde{g}_{n, l}\left(k_{*}\right) \sim\left(k_{*}\right)^{l} .
$$

Fig. 1 illustrates this behavior of functions $a_{l}^{1 s}\left(k_{*}, q_{*}\right)$. The functions $a_{0}^{1 s}\left(k_{*}, q_{*}\right), a_{1}^{1 s}\left(k_{*}, q_{*}\right), \nu_{0}^{1 s}\left(k_{*}, q_{*}\right)$, $\nu_{1}^{1 s}\left(k_{*}, q_{*}\right)$ are given in the analytical form in the Appendix.
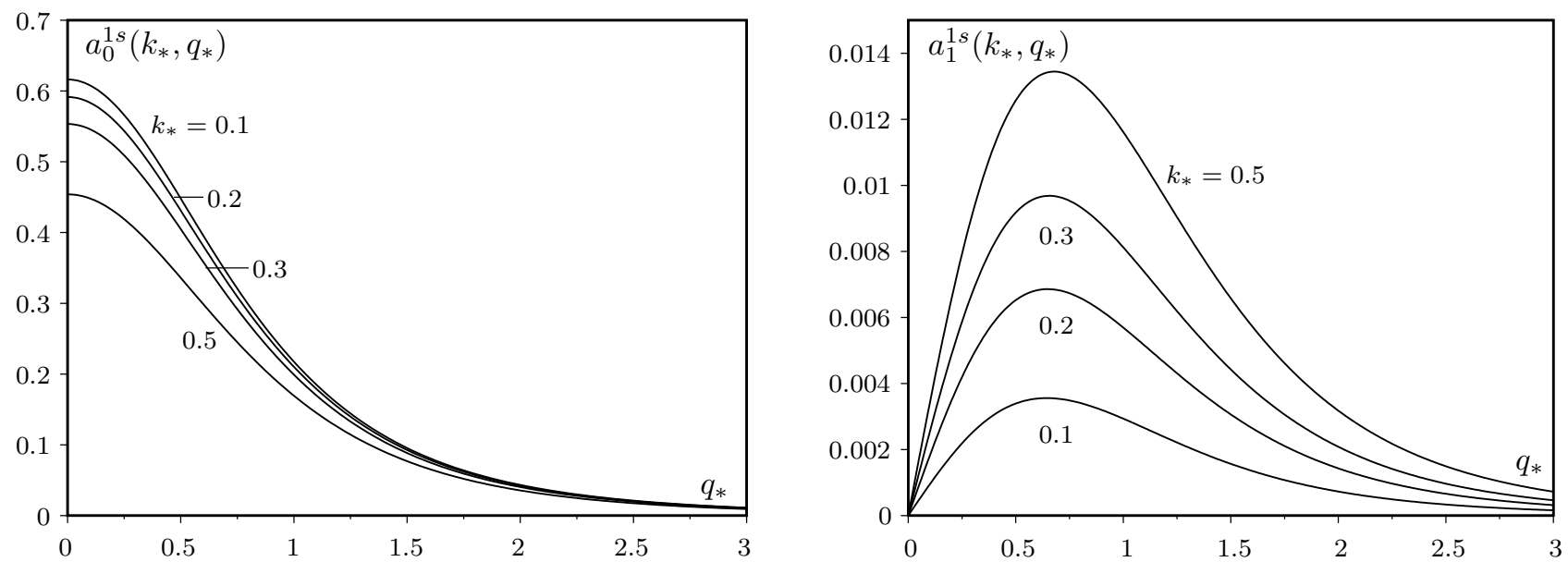

Fig. 1. Dependence of the functions $a_{0}^{1 s}\left(k_{*}, q_{*}\right)$ and $a_{1}^{1 s}\left(k_{*}, q_{*}\right)$ on the variable $q_{*}$ at different values $k_{*}$. 
To yield the asymptotics $F_{1 s}^{(1)}(\mathbf{k} \mid \mathbf{r})$ on the large distances from the proton, we replace the function $\varphi_{\mathbf{q}}(\mathbf{r})$ in the formula (35) on $\varphi_{\mathbf{q}}^{(0)}(\mathbf{r})$ and expand the latter one into the spherical harmonics. Integrating over the angular variables of vector $\mathbf{q}$ according to

$$
\int d \Omega_{q} P_{l}(\widehat{\mathbf{k}, \mathbf{q}}) P_{l_{1}}(\widehat{\mathbf{q}, \boldsymbol{\rho}})=\frac{4 \pi}{2 l+1} \delta_{l, l_{1}} P_{l}(\widehat{\mathbf{k}, \boldsymbol{\rho}}),
$$

we find following asymptotics $\chi_{1 s}(\mathbf{k} \mid \mathbf{r})$ :

$$
\begin{aligned}
\chi_{1 s}(\mathbf{k} \mid \mathbf{r}) & =-V^{1 / 2} \sum_{l=0}^{\infty}(2 l+1) P_{l}(\widehat{\mathbf{k}, \boldsymbol{\rho}}) T_{l}^{1 s}\left(k_{*} \mid \rho\right), \\
T_{l}^{1 s}\left(k_{*} \mid \rho\right) & =i^{l} \frac{4}{\pi} \int_{0}^{\infty} \frac{d q_{*} q_{*}^{2}}{q_{*}^{2}-k_{*}^{2}} j_{l}\left(q_{*} \rho\right) \Xi_{l}^{1 s}\left(k_{*}, q_{*}\right) .
\end{aligned}
$$

Taking into account the asymptotics of the Bessel functions [19]

$$
j_{l}(z) \approx \frac{1}{z} \sin \left[z-\frac{\pi}{2} l\right] \quad \text { at } \quad z \gg 1,
$$

as well as the expression

$$
\Xi_{l}^{1 s}\left(k_{*},-q_{*}\right)=(-1)^{l} \Xi_{l}^{1 s}\left(k_{*}, q_{*}\right),
$$

we reduce $T_{l}^{1 s}\left(k_{*} \mid \rho\right)$ to such form

$$
T_{l}^{1 s}\left(k_{*} \mid \rho\right)=[i \pi \rho]^{-1} \int_{-\infty}^{+\infty} \frac{d q_{*} q_{*}}{q_{*}^{2}-k_{*}^{2}} e^{i q_{*} \rho} \Xi_{l}^{1 s}\left(k_{*}, q_{*}\right) \approx \rho^{-1} e^{i k_{*} \rho} \Xi_{l}^{1 s}\left(k_{*}, k_{*}\right) .
$$

It follows that $F_{1 s}(\mathbf{k} \mid \mathbf{r})$ has the asymptotics

$$
F_{1 s}(\mathbf{k} \mid \mathbf{r}) \Rightarrow V^{-1 / 2}\left\{e^{i\left(\mathbf{k}_{*}, \boldsymbol{\rho}\right)}-\rho^{-1} e^{i k_{*} \rho} \sum_{l=0}^{\infty}(2 l+1) P_{l}(\widehat{\mathbf{k}, \boldsymbol{\rho}}) \Xi_{l}^{1 s}\left(k_{*}, k_{*}\right)\right\},
$$

which is the sum of the plane and spherical waves, and the expression

$$
S_{1 s}\left(k_{*}\right)=4 \pi \sum_{l=0}^{\infty}(2 l+1)\left[\Xi_{l}^{1 s}\left(k_{*}, k_{*}\right)\right]^{2} a_{0}^{2}
$$

determines the total cross-section of the scattering of the electron on the hydrogen atom, which is in ground state, taking into account electrostatic and exchange corrections.

The calculation of Born corrections $\chi_{\sigma_{1}}(\mathbf{k} \mid \mathbf{r})$ at $\sigma_{1} \neq 1 s$ are performed according to the scheme described above. In the case of $n s$-states $(n \geqslant 2)$ we get the analog of the expression (35)

$$
\chi_{n s}(\mathbf{k} \mid \mathbf{r})=-\frac{4 \pi e^{2} a_{0}^{2}}{V} \sum_{\mathbf{q}} \frac{\varphi_{\mathbf{q}}(\mathbf{r})}{\varepsilon_{q}-\varepsilon_{k}+\left(1-n^{-2}\right) e^{2} / 2 a_{0}} \sum_{l=0}^{\infty}(2 l+1) P_{l}\left(\mathbf{k}_{*}, \mathbf{q}_{*}\right) \Xi_{l}^{1 s, n s}\left(k_{*}, q_{*}\right) .
$$

Herewith

$$
\begin{aligned}
\Xi_{l}^{1 s, n s}\left(k_{*}, q_{*}\right)= & \left\{8 a_{l}^{1 s, n s}\left(k_{*}, q_{*}\right)+\omega_{l}^{1 s, n s}\left(k_{*}, q_{*}\right)\right\} \\
& -(-i)^{l} \sum_{n_{\sigma} \geqslant l+1} \tilde{g}_{n_{\sigma}, l}^{*}\left(k_{*}\right)\left\{8 b_{n_{\sigma}, l}^{1 s, n s}\left(q_{*}\right)+\omega_{n_{\sigma}, l}^{1 s, n s}\left(q_{*}\right)\right\},
\end{aligned}
$$

where introduced here functions are the analogous to corresponding expressions from the formula (37): $a_{l}^{1 s, n s}\left(k_{*}, q_{*}\right)$ and $b_{n_{\sigma}, l}^{1 s, n s}\left(k_{*}, q_{*}\right)$ which are formed from $a_{l}^{1 s}\left(k_{*}, q_{*}\right)$ and $b_{n, l}^{1 s}\left(k_{*}, q_{*}\right)$ by the replacement $\exp \left(-\rho_{2}\right) \rightarrow 1 / 2 R_{n, 0}\left(\rho_{2}\right) ; \omega_{l}^{1 s, n s}\left(k_{*}, q_{*}\right)$ and $\omega_{n_{\sigma}, l}^{1 s, n s}\left(q_{*}\right)$ we get from $\omega_{l}^{1 s}\left(k_{*}, q_{*}\right)$ and $\omega_{n, l}^{1 s}\left(q_{*}\right)$ by the replacement $\nu_{1 s}(\rho) \rightarrow \omega_{1 s, n s}(\rho)=\left(e^{2} / a_{0}\right)^{-1} \omega_{1 s, n s}(\mathbf{r})$; the expressions for $\tilde{g}_{n, l}\left(q_{*}\right)$ and $\tilde{g}_{n_{\sigma}, l}\left(q_{*}\right)$ coincide at $n=n_{\sigma}$. 
Integrating the expression (47) over the angular variables of the vector $\mathbf{q}$, we find the explicit expression for the function $\chi_{n s}(\mathbf{k} \mid \mathbf{r})$ :

$$
\begin{aligned}
\chi_{n s}(\mathbf{k} \mid \mathbf{r}) & =V^{1 / 2} \sum_{l=0}^{\infty}(i)^{l} P_{l}(\widehat{\mathbf{k}, \boldsymbol{\rho}}) T_{l}^{1 s, n s}\left(k_{*} \mid \rho\right), \\
T_{l}^{1 s, n s}\left(k_{*} \mid \rho\right) & =-\frac{4}{\pi} \int_{0}^{\infty} \frac{d q_{*} q_{*}^{2} j_{l}\left(q_{*} \rho\right)}{q_{*}^{2}+\left(1-n^{-2}\right)-k_{*}^{2}} \Xi_{l}^{1 s, n s}\left(k_{*}, q_{*}\right) .
\end{aligned}
$$
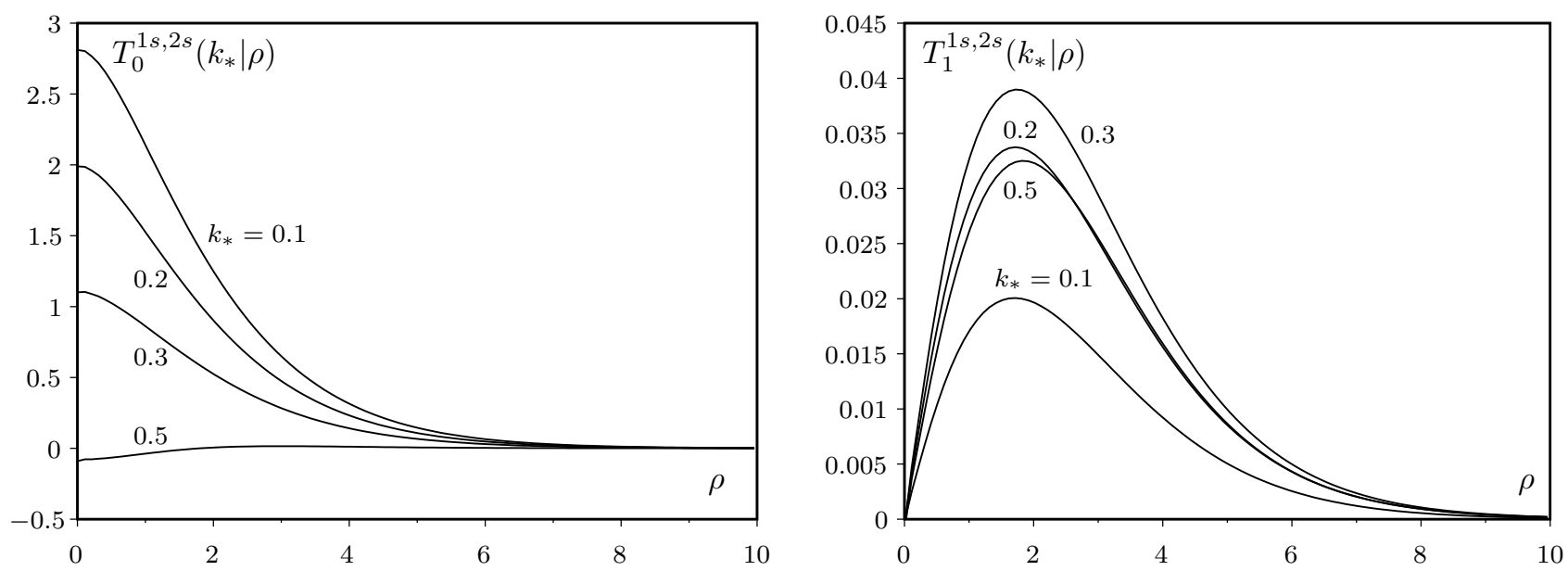

Fig. 2. Dependence of the functions $T_{0}^{1 s, 2 s}\left(k_{*} \mid \rho\right)$ and $T_{1}^{1 s, 2 s}\left(k_{*} \mid \rho\right)$ on the variable $\rho$ at different values $k_{*}$.

Unlike (41), the expression (49) determines the dependence $\chi_{n s}(\mathbf{k} \mid \mathbf{r})$ at the arbitrary values $\mathbf{r}$, and the integrand does not have singularity at $k_{*}^{2}<1-n^{-2}$. Therefore the functions $T_{l}^{1 s, n s}\left(k_{*} \mid \rho\right)$ are localized at small values of the photoelectron energy and resemble the hydrogen functions, which are illustrated in Fig. 2 with $T_{0}^{1 s, 2 s}\left(k_{*} \mid \rho\right)$ and $T_{1}^{1 s, 2 s}\left(k_{*} \mid \rho\right)$. Therefore, when taking into account the terms $\sigma_{1} \neq 1 s$ the wave function (19) has the character of a variational wave function [10] and this is different from the wave function of the system "atom + photoelectron" in the model with "hard" hydrogen atom. However the functions $T_{1}^{1 s, 2 s}\left(k_{*} \mid \rho\right)$ depend on the photoelectron energy, and in the region $k_{*}^{2}>1-n^{-2}$ the function $\chi_{n, s}(\mathbf{k} \mid \mathbf{r})$ is described by the spherical wave, as well as $\chi_{1, s}(\mathbf{k} \mid \mathbf{r})$, unlike the function of the work [10].

\subsection{The two-electron transitions at the one-electron photoionization of ion $\mathrm{H}^{-}$}

If the photon energy is in the interval

$$
\left(1-n^{-2}+0.0555\right) \frac{e^{2}}{2 a_{0}} \leqslant \hbar \omega<1.0555 \ldots \frac{e^{2}}{2 a_{0}},
$$

then it becomes possible the process of one-electron photoionization, the final result of which will be neutral atom in the excited state and photoelectron with the energy $\varepsilon_{k}=\hbar \omega-\left(\varepsilon_{\text {exc }}\right) e^{2} / 2 a_{0}$. We consider here the scheme of calculation of the wave function of the system "excited atom + photoelectron", when after the collision the atomic electron is in the state with the wave function $\varphi_{\sigma}(\mathbf{r})=R_{n, l}(\rho) Y_{l, m}(\theta, \varphi) a_{0}^{-3 / 2}$, where $l=0 ; 1$. In the model of "hard" atom the two-electron wave function

$$
\Psi_{\sigma, \mathbf{k}}\left(\mathbf{r}_{1}, \mathbf{r}_{2}\right)=\frac{1}{\sqrt{2}}\left\{\varphi_{\sigma}\left(\mathbf{r}_{1}\right) F_{\sigma}\left(\mathbf{k} \mid \mathbf{r}_{2}\right)+\varphi_{\sigma}\left(\mathbf{r}_{2}\right) F_{\sigma}\left(\mathbf{k} \mid \mathbf{r}_{1}\right)\right\},
$$

and the function $F_{\sigma}(\mathbf{r})$ is the solution of equation

$$
\left\{-\frac{\hbar^{2} \nabla^{2}}{2 m}-\varepsilon_{k}+\nu_{\sigma}(r)\right\} F_{\sigma}(\mathbf{k} \mid \mathbf{r})+\varphi_{\sigma}(\mathbf{r}) \int d \mathbf{r}_{2} \varphi_{\sigma}^{*}\left(\mathbf{r}_{2}\right) \frac{e^{2}}{\left|\mathbf{r}-\mathbf{r}_{2}\right|} F_{\sigma}\left(\mathbf{k} \mid \mathbf{r}_{2}\right)=0 .
$$

Mathematical Modeling and Computing, Vol. 7, No. 1, pp. 125-139 (2020) 
In the first Born approximation

$$
\begin{aligned}
F_{\sigma}^{(1)}(\mathbf{k} \mid \mathbf{r}) & =\varphi_{\mathbf{k}}(\mathbf{r})+\chi_{\sigma}(\mathbf{k} \mid \mathbf{r}), \\
\chi_{\sigma}(\mathbf{k} \mid \mathbf{r}) & =-\int G_{\sigma}\left(\mathbf{r}, \mathbf{r}^{\prime}\right)\left\{\nu_{\sigma}\left(\mathbf{r}^{\prime}\right) \varphi_{\mathbf{k}}\left(\mathbf{r}^{\prime}\right)+\varphi_{\sigma}\left(\mathbf{r}^{\prime}\right) \int d \mathbf{r}_{2} \varphi_{\sigma}^{*}\left(\mathbf{r}_{2}\right) \frac{e^{2}}{\left|\mathbf{r}^{\prime}-\mathbf{r}_{2}\right|} \varphi_{\mathbf{k}}\left(\mathbf{r}_{2}\right)\right\} d \mathbf{r}^{\prime}, \\
G_{\sigma}\left(\mathbf{r}, \mathbf{r}^{\prime}\right) & =\frac{1}{V} \sum_{\mathbf{q}} \varphi_{q}(\mathbf{r}) \exp \left[-i\left(\mathbf{q}, \mathbf{r}^{\prime}\right)\right]\left\{\varepsilon_{q}-\varepsilon_{k}\right\}^{-1} ; \\
\nu_{\sigma}(\mathbf{r}) & =-\frac{e^{2}}{r}+\int d \mathbf{r}_{2}\left|\varphi_{\sigma}\left(\mathbf{r}_{2}\right)\right|^{2} e^{2}\left|\mathbf{r}-\mathbf{r}_{2}\right|^{-1}=-\frac{e^{2}}{a_{0}} \nu_{\sigma}(\rho) .
\end{aligned}
$$

We perform the calculation of cross-sections for $n s$-states because the cross-sections for $n p$-states are negligible. In particular, in the case of $2 s$-state we obtain the analog of the expression (35),

$$
\begin{aligned}
\chi_{2 s}(\mathbf{k} \mid \mathbf{r}) & =-\frac{4 \pi e^{2} a_{0}^{2}}{V} \sum_{\mathbf{q}} \frac{\varphi_{q}(\mathbf{r})}{\varepsilon_{q}-\varepsilon_{k}} \sum_{l=0}(2 l+1) P_{l}(\widehat{\mathbf{k}, \mathbf{q}}) \Xi_{l}^{2 s}\left(k_{*}, q_{*}\right), \\
\Xi_{l}^{2 s}\left(k_{*}, q_{*}\right) & =8 a_{l}^{2 s}\left(k_{*}, q_{*}\right)+\nu_{l}^{2 s}\left(k_{*}, q_{*}\right)+\ldots ; \\
a_{l}^{2 s}\left(k_{*}, q_{*}\right) & =[8(2 l+1)]^{-1} \iint_{0}^{\infty} d \rho_{1} d \rho_{2}\left(\rho_{1} \rho_{2}\right)^{2} R_{2,0}\left(\rho_{1}\right) R_{2,0}\left(\rho_{2}\right) a_{l}\left(\rho_{1}, \rho_{2}\right) j_{l}\left(q \rho_{1}\right) j_{l}\left(k \rho_{2}\right) ; \\
\nu_{l}^{2 s}\left(k_{*}, q_{*}\right) & =\int_{0}^{\infty} d \rho \rho^{2} \nu_{2 s}(\rho) j_{l}\left(k_{*} \rho\right) j_{l}\left(q_{*} \rho\right) ; \\
\nu_{2 s}(\rho) & =-\frac{e^{-\rho}}{\rho}\left\{1+\frac{3}{4} \rho+\frac{\rho^{2}}{4}+\frac{\rho^{3}}{8}\right\} ; \ldots,
\end{aligned}
$$

where $R_{2,0}(\rho)$ are the radial functions of $2 s$ state.

\section{Matrix elements of momentum operators and photoionization cross-sections}

Let us consider the channel of photoionization, result of which is the hydrogen atom in the ground state, considering the main term of the function (19), when $\sigma=1 s$. In this approximation

$$
\mathbf{P}_{1 s}(\mathbf{k})=-\frac{i \hbar}{\sqrt{2}}\left\langle\Psi_{-}\left(\mathbf{r}_{1}, \mathbf{r}_{2}\right) \mid \varphi_{1 s}\left(\mathbf{r}_{2}\right)\left[\nabla_{1} \varphi_{\mathbf{k}}\left(\mathbf{r}_{1}\right)+\nabla_{1} \chi_{1 s}(\mathbf{k} \mid 1)\right]+\left[\varphi_{\mathbf{k}}\left(\mathbf{r}_{2}\right)+\chi_{1 s}\left(\mathbf{k} \mid \mathbf{r}_{2}\right)\right] \nabla_{1} \varphi_{1 s}\left(\mathbf{r}_{1}\right)\right\rangle,
$$

which gives 4 components of the vector $\mathbf{P}_{1 s}(\mathbf{k})$. Separating out the dimensional factor, we write $\mathbf{P}_{1 s}(\mathbf{k})$ in the form

$$
\mathbf{P}_{1 s}(\mathbf{k})=\frac{\hbar}{a_{0}} \cdot \frac{\mathbf{k}}{k}\left(\frac{a_{0}^{3}}{V}\right)^{1 / 2} \sum_{i=1}^{4} P_{i}\left(k_{*}\right),
$$

where $P_{i}\left(k_{*}\right)$ are the dimensionless functions of the variable $k_{*}$, namely

$$
\begin{aligned}
& P_{1}\left(k_{*}\right)=32 a_{1} N_{1} \pi^{1 / 2}\left(\alpha_{1} \alpha_{2}\right)^{3 / 2} k_{*}\left\{\frac{\alpha_{1}}{\left(1+\alpha_{2}\right)^{3}\left(\alpha_{1}^{2}+k_{*}^{2}\right)^{2}}+\frac{\alpha_{2}}{\left(1+\alpha_{1}\right)^{3}\left(\alpha_{2}^{2}+k_{*}^{2}\right)^{2}}\right\} ; \\
& P_{2}\left(k_{*}\right)=-\frac{128}{3^{1 / 2}}(2 \pi)^{1 / 2} a_{2} \frac{\gamma_{2}^{6}}{\left(1+\gamma_{2}\right)^{4}} \cdot \frac{k_{*}}{\left.\left(\gamma_{2}^{2}\right)+k_{*}^{2}\right)^{3}} ; \\
& P_{3}\left(k_{*}\right)=-a_{1} N_{1}\left(\alpha_{1} \alpha_{2}\right)^{3 / 2} \frac{128}{\pi^{1 / 2}} \int_{0}^{\infty} \frac{d q_{*} q_{*}^{3}}{q_{*}^{2}-k_{*}^{2}} \Xi_{1}^{1 s}\left(k_{*}, q_{*}\right)\left\{\frac{\alpha_{1}}{\left(1+\alpha_{2}\right)^{3}\left(\alpha_{1}^{2}+q_{*}^{2}\right)^{2}}+\frac{\alpha_{2}}{\left(1+\alpha_{1}\right)^{3}\left(\alpha_{2}^{2}+q_{*}^{2}\right)^{2}}\right\} ; \\
& P_{4}\left(k_{*}\right)=a_{2} \frac{128}{\pi^{1 / 2}} \cdot \frac{8}{\sqrt{6}} \cdot \frac{\gamma_{2}^{6}}{\left(1+\gamma_{2}\right)^{4}} \int_{0}^{\infty} \frac{d q_{*} q_{*}^{3}}{q_{*}^{2}-k_{*}^{2}} \Xi_{1}^{1 s}\left(k_{*}, q_{*}\right) \frac{1}{\left(\gamma_{2}^{2}+q_{*}^{2}\right)^{3}} .
\end{aligned}
$$

Mathematical Modeling and Computing, Vol. 7, No.1, pp. 125-139 (2020) 
Calculated expression for $\mathbf{P}_{1 s}(\mathbf{k})$ can be obtained if instead of the function $F_{1 s}(\mathbf{k} \mid \mathbf{r})$ used only its $p$-wave component

$$
F_{1 s}^{(p)}(\mathbf{k} \mid \mathbf{r})=\frac{3 i}{\sqrt{V}}\left\{j_{1}\left(k_{*} r\right) P_{1}(\hat{\mathbf{k}, \mathbf{r}})-\frac{4 \pi e^{2} a_{0}^{2}}{V} \sum_{\mathbf{q}} j_{1}\left(q_{*} r\right) P_{1}(\hat{\mathbf{q}, \hat{r}}) P_{1}\left(k_{*}, q_{*}\right) \Xi_{1}^{1 s}\left(k_{*}, q_{*}\right)\right\},
$$

which has the structure of main terms of the variational wave functions from the work [10]. The terms $P_{1}\left(k_{*}\right)$ and $P_{2}\left(k_{*}\right)$ correspond to the plane wave approximation for the function $F_{1 s}(\mathbf{k} \mid \mathbf{r})$. The terms $P_{3}\left(k_{*}\right)$ and $P_{4}\left(k_{*}\right)$ created by the Born correction are small in modulus and make a small contribution to the cross-section.

We have also taken into account the term

$$
\frac{1}{\sqrt{2}}\left\{\varphi_{2 s}\left(\mathbf{r}_{1}\right) \chi_{2 s}\left(\mathbf{k} \mid \mathbf{r}_{2}\right)+\varphi_{2 s}\left(\mathbf{r}_{2}\right) \chi_{2 s}\left(\mathbf{k} \mid \mathbf{r}_{1}\right)\right\}
$$

in the function (19), where $\chi_{2 s}(\mathbf{k} \mid \mathbf{r})$ are determined by the expressions (47), (48), which give the dimensionless matrix elements

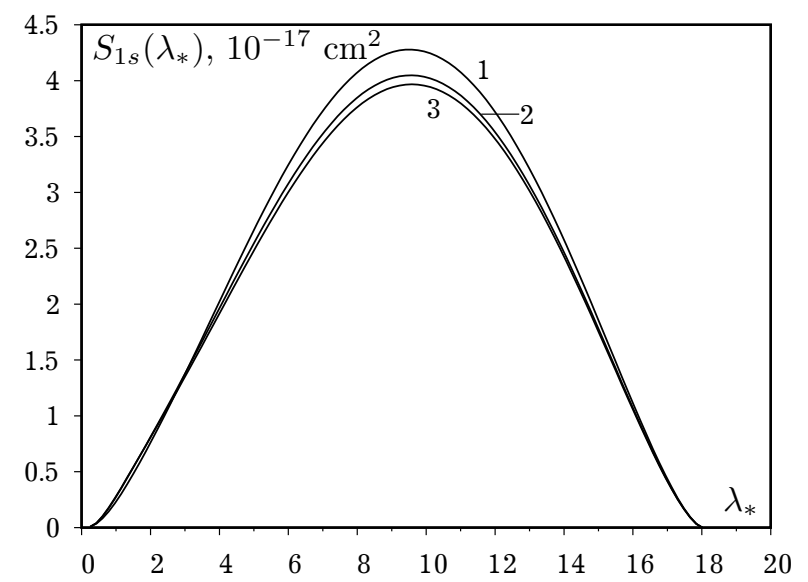

Fig. 3. Dependence of the cross-section $S_{1 s}\left(\lambda_{*}\right)$ on the wavelength. Curve 1 takes into account the terms $P_{1}\left(k_{*}\right)$ and $P_{2}\left(k_{*}\right)$; curve $2-P_{1}\left(k_{*}\right)-P_{3}\left(k_{*}\right)$; curve 3 accounts for $P_{1}\left(k_{*}\right)-P_{6}\left(k_{*}\right)$.

$$
\begin{aligned}
P_{5}\left(k_{*}\right)= & -\frac{16}{\sqrt{2 \pi}} a_{1} N_{1}\left(\alpha_{1} \alpha_{2}\right)^{3 / 2} \int_{0}^{\infty} \frac{d q_{*} q_{*}^{3}}{q_{*}^{2}-k_{*}^{2}+3 / 4} \Xi_{1}^{1 s, 2 s}\left(k_{*}, q_{*}\right) \\
& \times\left\{\frac{\alpha_{1}\left(\alpha_{2}-1\right)}{\left(\alpha_{1}^{2}+q^{2}\right)^{2}\left(\alpha_{2}+1 / 2\right)^{4}}+\frac{\alpha_{2}\left(\alpha_{1}-1\right)}{\left(\alpha_{2}^{2}+q^{2}\right)^{2}\left(\alpha_{1}+1 / 2\right)^{4}}\right\} ; \\
P_{6}\left(k_{*}\right)= & \frac{64}{3 \sqrt{3 \pi}} a_{2} \frac{\gamma_{2}^{6}}{\left(\gamma_{2}+1 / 2\right)^{5}}\left(\gamma_{2}-1 / 2\right) \int_{0}^{\infty} \frac{d q_{*} q_{*}^{3}}{q_{*}^{2}-k_{*}^{2}+3 / 4} \Xi_{1}^{1 s, 2 s}\left(k_{*}, q_{*}\right) \frac{1}{\left(\gamma_{2}^{2}+q_{*}^{2}\right)^{3}} .
\end{aligned}
$$

At the calculation of the matrix elements of momentum operator were used the expansion coefficients $a_{1}, a_{2}$ and variational parameters $\alpha_{1}, \alpha_{2}$ and $\gamma_{2}$, given in (12).

According to the formula (4) the cross-section for the process of photoionization in $1 s$-channel in the wavelength scale is

$$
S_{1 s}(\omega)=\frac{16}{3} \alpha_{0} a_{0}^{2} \frac{k_{*}\left(\omega_{*}\right)}{\omega_{*}}\left|\sum_{i=1}^{6} P_{i}\left(k_{*}\left(\omega_{*}\right)\right)\right|^{2}
$$

where $k_{*}\left(\omega_{*}\right)=\left(\omega_{*}-\Delta \varepsilon\right)^{1 / 2}=\left(1 / \lambda_{*}-\Delta \varepsilon\right)^{1 / 2}$, $\alpha_{0}=e^{2} / \hbar c$ is the fine structure constant. The role of individual terms $P_{i}\left(k_{*}\right)$ is shown in Fig. 3, in which the cross-section $S_{1 s}$ is depicted in the scale of wavelengths in the units $10^{-17} \mathrm{~cm}^{2}$. It was also depicted the result of calculation from the work [10]. The result of this cross-section was shown in Table 1 in the wavelength scale and dimensionless wave vector, according to the formula (61).

Table 1. The cross-section $S_{1 s}$ in the units $10^{-17} \mathrm{~cm}^{2}$, which calculated by the expressions (56), $(57),(60),(61)$ in the scale of dimensionless wave vectors $k_{*}$ and the dimensionless wavelength $\lambda_{*}$.

\begin{tabular}{|c|c|c||c|c|c|}
\hline$k_{*}$ & $\lambda_{*}$ & $S_{1 s}$ & $k_{*}$ & $\lambda_{*}$ & $S_{1 s}$ \\
\hline 0.01 & 17.9856 & 0.0025 & 0.29 & 7.1633 & 3.5032 \\
\hline 0.05 & 17.2414 & 0.2810 & 0.30 & 6.8729 & 3.3918 \\
\hline 0.10 & 15.2672 & 1.5678 & 0.35 & 5.6180 & 2.8101 \\
\hline 0.15 & 12.8205 & 3.1063 & 0.40 & 4.6404 & 2.2795 \\
\hline 0.20 & 10.4712 & 3.9002 & 0.45 & 3.8760 & 1.8450 \\
\hline 0.21 & 10.0402 & 3.9492 & 0.50 & 3.2733 & 1.5030 \\
\hline 0.22 & 9.6246 & 3.9668 & 0.55 & 2.7933 & 1.2355 \\
\hline 0.23 & 9.2251 & 3.9563 & 0.60 & 2.4067 & 1.0244 \\
\hline 0.24 & 8.8417 & 3.9215 & 0.65 & 2.0921 & 0.8553 \\
\hline 0.25 & 8.4746 & 3.8659 & 0.70 & 1.8332 & 0.7175 \\
\hline 0.26 & 8.1235 & 3.7931 & 0.75 & 1.6181 & 0.6037 \\
\hline 0.27 & 7.7882 & 3.7064 & 0.80 & 1.4378 & 0.5085 \\
\hline 0.28 & 7.4683 & 3.6088 & 0.85 & 1.2854 & 0.4284 \\
\hline
\end{tabular}

For the calculation of the cross-section of photoionization of $\mathrm{H}^{-}$ion, when is formed the excited hydrogen atom, we used the wave functions (51)-(53). In particular in the case $\sigma=2 s$ the function (54) 
gives the cross-section, which determined by the formula (61), in which $k_{*}\left(\omega_{*}\right)=\left\{\omega_{*}-\Delta \varepsilon-3 / 4\right\}^{1 / 2}$, and the dimensionless matrix elements are as follows:

$$
\begin{aligned}
P_{1}^{2 s}\left(k_{*}\right) & =16 \sqrt{2 \pi} a_{1} N_{1}\left(\alpha_{1} \alpha_{2}\right)^{3 / 2} k_{*} \mu\left(\alpha_{1}, \alpha_{2} \mid k_{*}\right) \\
P_{2}^{2 s}\left(k_{*}\right) & =-128 \sqrt{6 \pi} a_{2} \gamma_{2}^{6} \frac{\left(\gamma_{2}-1 / 2\right)}{\left(\gamma_{2}+1 / 2\right)^{5}} \frac{k_{*}}{\left(\gamma_{2}^{2}+1 / 2\right)^{3}} ; \\
P_{3}^{2 s}\left(k_{*}\right) & =-\frac{64}{\sqrt{2 \pi}} a_{1} N_{1}\left(\alpha_{1} \alpha_{2}\right)^{3 / 2} \int_{0}^{\infty} \frac{d q_{*} q_{*}^{3}}{q_{*}^{2}-k_{*}^{2}} \Xi_{1}^{2 s}\left(k_{*}, q_{*}\right) \mu\left(\alpha_{1}, \alpha_{2} \mid q_{*}\right) ; \\
P_{4}^{2 s}\left(k_{*}\right) & =\frac{256}{\sqrt{3 \pi}} \gamma_{2}^{6} a_{2}\left(\gamma_{2}-\frac{1}{2}\right) k_{*} \frac{1}{\left(\gamma_{2}+1 / 2\right)^{5}} \int_{0}^{\infty} \frac{d q_{*} q_{*}^{2}}{q_{*}^{2}-k_{*}^{2}} \Xi_{1}^{2 s}\left(k_{*}, q_{*}\right) \frac{1}{\left(\gamma_{2}^{2}+q_{*}^{2}\right)^{3}} ; \\
\mu\left(\alpha_{1}, \alpha_{2} \mid x\right) & =\left\{\frac{\left(\alpha_{2}-1\right) \alpha_{1}}{\left(\alpha^{2}+1 / 2\right)^{4}\left(\alpha_{1}^{2}+x^{2}\right)^{2}}+\frac{\left(\alpha_{1}-1\right) \alpha_{2}}{\left(\alpha_{1}+1 / 2\right)^{4}\left(\alpha_{2}^{2}+x^{2}\right)^{2}}\right\} .
\end{aligned}
$$

As was shown in Fig. 4 , the cross-section $S_{2 s}\left(\lambda_{*}\right)$ is non-zero in the range $0 \leqslant \lambda_{*} \leqslant 1.2415$, and its maximum is approximately in 13 times less than the cross-section maximum of $S_{1 s}\left(\lambda_{*}\right)$.

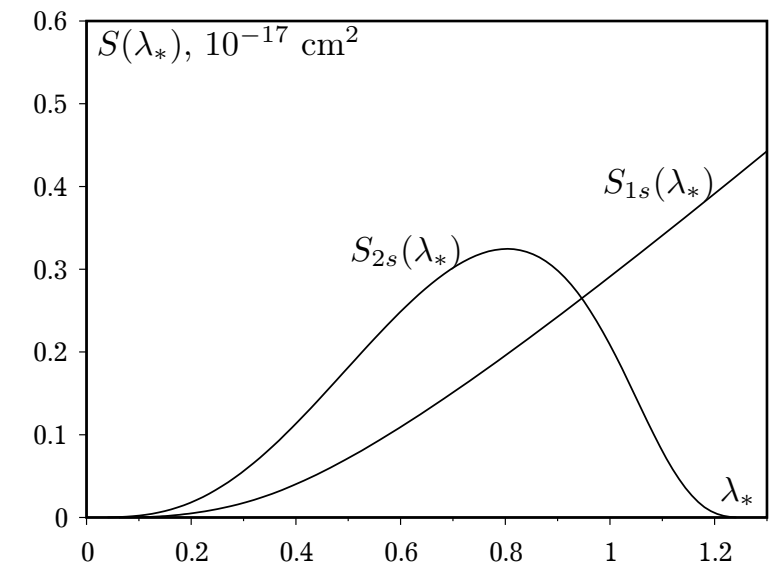

Fig. 4. Dependence of the cross-sections $S_{1 s}\left(\lambda_{*}\right)$ and $S_{2 s}\left(\lambda_{*}\right)$ on wavelength.

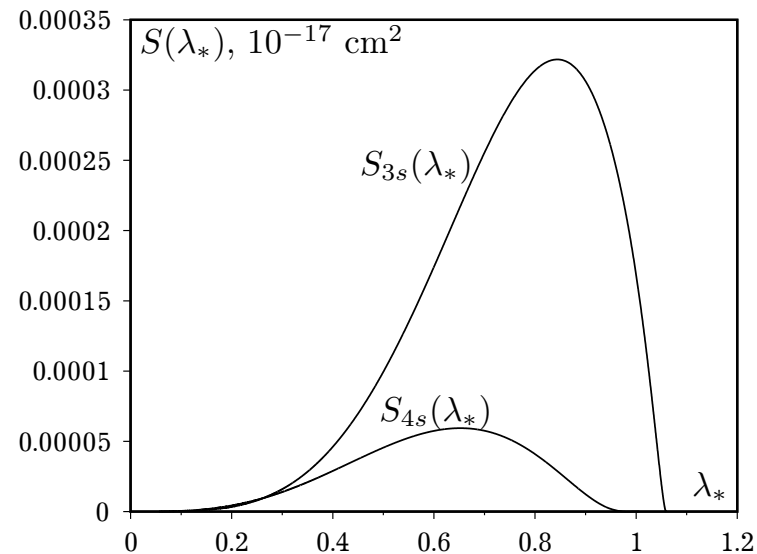

Fig. 5. Dependence of the cross-sections $S_{3 s}\left(\lambda_{*}\right)$ and $S_{4 s}\left(\lambda_{*}\right)$ on wavelength.

The cross-section $S_{2 p}\left(\lambda_{*}\right)$ is very small, which was shown from the plane wave contribution to the matrix element of momentum operator

$$
\mathbf{P}_{2,1,0}(\mathbf{k})=\left\{\frac{\hbar}{a_{0}} \frac{\mathbf{k}}{k}\left(\frac{a_{0}^{3}}{V}\right)^{1 / 2}\right\} i \frac{256 \pi a_{2}}{3\left(\gamma_{2}+k^{2}\right)^{3}} \frac{\gamma_{2}^{6}}{\left(\gamma_{2}+\frac{1}{2}\right)^{5}} k_{*}^{2} Y_{1,0}(\mathbf{k}) .
$$

Because of the matrix operator is imaginary, its contribution to the dimensionless cross-section (in the units $10^{-17} \mathrm{~cm}^{2}$ ) has the order $10^{-5}$. In the case $3 s$ - and $4 s$-states in the plane wave approximation

$$
\begin{aligned}
P_{1}^{3 s}\left(k_{*}\right)= & \frac{32 \sqrt{\pi}}{3 \sqrt{3}} a_{1} N_{1}\left(\alpha_{1}, \alpha_{2}\right)^{3 / 2} k_{*} \\
& \times\left\{\frac{\alpha_{1}}{\left(\alpha_{1}^{2}+k_{*}^{2}\right)^{2}} \frac{\alpha_{2}^{2}-4 / 3 \alpha_{2}+1 / 2}{\left(\alpha_{2}+1 / 3\right)^{5}}+\frac{\alpha_{2}}{\left(\alpha_{2}^{2}+k_{*}^{2}\right)^{2}} \frac{\alpha_{1}^{2}-4 / 3 \alpha_{1}+1 / 3}{\left(\alpha_{1}+1 / 3\right)^{5}}\right\} ; \\
P_{2}^{3 s}\left(k_{*}\right)= & -\frac{256 \sqrt{\pi}}{27 \sqrt{2}} a_{2} k_{*} \frac{\gamma_{2}^{6}}{\left(\gamma_{2}+1 / 3\right)^{6}} \frac{3 k_{*}^{2}-22 / 9 \gamma_{2}+1 / 3}{\left(\gamma_{2}^{2}+k_{*}^{2}\right)^{3}} ; \\
P_{1}^{4 s}\left(k_{*}\right)= & 4 a_{1} N_{1}\left(\alpha_{1} \alpha_{2}\right)^{3 / 2} \sqrt{\pi} k_{*} \\
& \times\left\{\frac{\alpha_{1}}{\left(\alpha_{1}^{2}+k_{*}^{2}\right)^{2}} \frac{\alpha_{2}^{2}-3 / 2 \alpha_{2}^{2}+9 / 16 \alpha_{2}-1 / 16}{\left(\alpha_{2}+1 / 4\right)^{6}}+\frac{\alpha_{2}}{\left(\alpha_{2}^{2}+k_{*}^{2}\right)^{2}} \frac{\alpha_{1}^{2}-4 / 3 \alpha_{1}+1 / 3}{\left(\alpha_{1}+1 / 3\right)^{5}}\right\}
\end{aligned}
$$

The spectral dependence of the cross-sections $S_{3 s}(\lambda)$ and $S_{4 s}(\lambda)$ is shown in Fig. 5 . 


\section{Conclusions}

Our approach is based on the calculation of two electron wave function of the system "hydrogen atom + photoelectron" with help of the perturbation theory, namely in the Born approximation. Its applicability is due not only accounting for the orthogonalization effects, but also because the calculation of matrix elements of momentum operator in dipole approximation does not contain whole the wave function $F_{1 s}(\mathbf{k} \mid \mathbf{r})$, but its so called $p$-wave part, which corresponds to the partial wave with orbital number $l=1$ and is small (see. (58)).

As was shown from the calculation of the cross-section, $S_{1 s}\left(\lambda_{*}\right)$ according to the expression (61), the approximation of "hard" atom is principal, and taking into account the terms of type $\left\{\varphi_{\sigma_{1}}\left(\mathbf{r}_{1}\right) \chi_{\sigma_{1}}\left(\mathbf{k} \mid \mathbf{r}_{2}\right)+\right.$ $\left.\varphi_{\sigma_{1}}\left(\mathbf{r}_{2}\right) \chi_{\sigma_{1}}\left(\mathbf{k} \mid \mathbf{r}_{1}\right)\right\}$ gives small corrections, which are caused by the polarization of atom by photoelectron. Herewith $\chi_{2 s}(\mathbf{k} \mid \mathbf{r})$ are the localized functions in the region of photoelectron energy $\varepsilon_{k}<\frac{3}{4}$, that describe the change of electron distribution structure in the atom.

Concerning the cross-section of the photoionization process with excited atom, the

Table 2. The photoionization cross-section of the $H^{-}$ion in the units $10^{-17} \mathrm{~cm}^{2}$ as the function $k_{*}$ (approximation for the results of S. Geltman, [10]; our results, [*]).

\begin{tabular}{|c|c|c|c|c|c|}
\hline$k_{*}$ & {$[10]$} & {$[11]$} & {$[13]$} & {$[14]$} & {$[*]$} \\
\hline 0.1000 & 1.5202 & - & 1.5650 & 1.5530 & 1.5678 \\
\hline 0.1414 & 2.8100 & 2.7908 & 2.8620 & 2.8460 & 2.8781 \\
\hline 0.2000 & 3.8470 & 3.9820 & 3.9120 & 3.8980 & 3.9002 \\
\hline 0.2100 & 3.9080 & - & - & - & 3.9492 \\
\hline 0.2200 & 3.9388 & - & - & - & 3.9668 \\
\hline 0.2300 & 3.9412 & - & - & - & 3.9563 \\
\hline 0.2400 & 3.9211 & - & - & - & 3.9215 \\
\hline 0.2449 & 3.9029 & 3.9560 & 3.9780 & 3.9650 & 3.8966 \\
\hline 0.2828 & 3.6314 & 3.7590 & 3.7170 & 3.7080 & 3.5800 \\
\hline 0.3162 & 3.2846 & 3.3130 & 3.3730 & 3.3680 & 3.2042 \\
\hline
\end{tabular}

most significant is the cross-section for the atom in the state $2 s$. Other cross-sections are negligibly small.

As was shown from Table 2, the cross-section $S_{1 s}\left(k_{*}\right)$ calculated by us is close to the results of works $[10,11,13,14]$. We obtained the value $S_{1 s}^{(\max )}\left(k_{*}\right)=3.9668$ at $k_{*}=0.22$. This gives reasons for arguing, that the maximal cross-section value is in the limits

$$
3.96 \ldots \leqslant S_{1 s}^{(\max )} \cdot 10^{17} \mathrm{~cm}^{-2} \leqslant 3.97 \ldots
$$

[1] Wildt R. Negative ions of hydrogen and the opacity of stellar atmospheres. Astrophysical Journal. 90, 611-620 (1939).

[2] Wildt R. The continuous spectrum of Stellar atmoospheres consisting only of atoms and negative ions of hydrogen. Astrophysical Journal. 93, 47-51 (1941).

[3] Chandrasekhar S. On the continuous absorption coefficient of the negative hydrogen ion. II. Astrophysical Journal. 102, 395-401 (1945).

[4] Chandrasekhar S., Breen F. H. On the continuous absorption coefficient of the negative hydrogen ion. III. Astrophysical Journal. 104, 430-445 (1946).

[5] John T. L., Seaton M. J. The photodetachment of $H^{-}$. Monthly Notices of the Royal Astronomical Society. 121 (1), 41-47 (1960).

[6] Smith S. J., Burch D. S. Relative Measurement of the Photodetachment cross-section for $H^{-}$. Phys. Rev. 116 (5) 1125-1131 (1959).

[7] Vavrukh M. V., Vasil'eva I. E., Stelmakh O. M., Tyshko N. L. Continuous Absorption and Depression in the Solar Spectrum at Wavelengths from 650 to $820 \mathrm{~nm}$. Kinemat. Phys. Celest. Bodies. 32 (3), 129-144 (2016).

[8] Neckel H., Labs D. The solar radiation between 3300 and 12500 A. Solar Physics. 90 (2), 205-258 (1984).

[9] Burlov-Vasil'ev K. A., Vasil'eva I. E., Matveev Yu. B. New measurments of the absolute spectral energy distribution of Solar radiations in the range $\lambda \lambda 650-1070 \mathrm{~nm}$. Kinematics and Physics of Celestial Bodies. 12 (3), 75-91 (1996). 
[10] Geltman S. The bound-free absorption coefficient of the hydrogen negative ion. Astrophysical Journal. 136 (3), 935-945 (1962).

[11] Broad J. T. One- and two-electron photoejection from $H^{-}$: A multichannel J-matrix calculation. Phys. Rev. A. 14 (6), 2159-2173 (1976).

[12] Vavrukh M. V., Stelmakh O. M. The cross-sections of the main processes that forms the continuous absorption coefficient in the photosphere of Sun-like stars. Journal of Physical Studies. 17 (4), 4902 (2013).

[13] Stewart A. L. A perturbation-variation study of photodetachment from $H^{-}$. J. Phys. B: Atom. Molec. Phys. 11 (22), 3851-3860 (1978).

[14] Wishart A. W. The bound-free photodetachment cross-section of $H^{-}$. J. Phys. B: Atom. Molec. Phys. 12 (21), 3511-3519 (1979).

[15] Tweed R. J. Correlated wavefunctions for helium-like atomic systems. J. Phys. B. 5 (4), $810-819$ (1972).

[16] Hart J. F., Herzberg G. Twenty-Parameter Eigenfunction and Energy Values of the Ground States of the He and He-Like ions. Phys. Rev. 106 (1), 79-82 (1957).

[17] Pekeris C. L. $1^{1} S, 2^{1} S$ and $2^{3} S$ States of $H^{-}$and of He. Phys. Rev. 126 (4), 1470-1476 (1962).

[18] Vavrukh M. V., Kostrobij P. P., Markovych B. M. Reference system approach in the theory of many-electrons systems. Rastr-7, Lviv (2017), (in Ukrainian).

[19] Abramowitz M., Stegun I. A. Handbook of Mathematical Functions With Formulas, Graphs, and Mathematical Tables. Government Printing Office: Washington (1972).

\section{Appendix}

The functions $a_{0}^{1 s}\left(k_{*}, q_{*}\right), a_{1}^{1 s}\left(k_{*}, q_{*}\right), \nu_{0}^{1 s}\left(k_{*}, q_{*}\right), \nu_{1}^{1 s}\left(k_{*}, q_{*}\right)$ are determined by such expressions:

$$
\begin{aligned}
& a_{0}^{1 s}\left(k_{*}, q_{*}\right)=\left\{10+k_{*}^{2}+q_{*}^{2}\right\}\left\{1+q_{*}^{2}\right\}^{-1}\left\{1+k_{*}^{2}\right\}^{-1}\left\{\left[4+k_{*}^{2}+q_{*}^{2}\right]^{2}-4 k_{*}^{2} q_{*}^{2}\right\}^{-1} \\
& a_{1}^{1 s}\left(k_{*}, q_{*}\right)=\left\{\left(3\left(q_{*}^{4}-2 q_{*}^{2}-\frac{1}{3}\right) k_{*}^{6}+3\left(q_{*}^{6}+6 q_{*}^{4}-3 q_{*}^{2}\right) k_{*}^{4}\right.\right. \\
& \left.+3\left(-2 q_{*}^{6}-3 q_{*}^{4}-12 q_{*}^{2}-3\right) k_{*}^{2}-q_{*}^{2}\left(q_{*}^{4}+9\right)-2\right) \ln \frac{\left(k_{*}-q_{*}\right)^{2}+4}{\left(k_{*}+q_{*}\right)^{2}+4} \\
& +16\left(k_{*}-q_{*}\right)\left(k_{*} q_{*}-1\right)\left[k_{*}^{4} q_{*}^{2}+\left(q_{*}^{3}+q_{*}\right)\left(k_{*}^{3}+k_{*}\right)+\left(q_{*}^{4}+4 q_{*}^{2}+1\right) k_{*}^{2}+q_{*}^{2}\right] \\
& \times \arctan \left(\frac{k_{*}}{2}-\frac{q_{*}}{2}\right)+16\left(k_{*}+q_{*}\right)\left(k_{*} q_{*}+1\right)\left[k_{*}^{4} q_{*}^{2}+\left(-q_{*}^{3}-q_{*}\right)\left(k_{*}^{3}+k_{*}\right)\right. \\
& \left.+\left(q_{*}^{4}+4 q_{*}^{2}+1\right) k_{*}^{2}+q_{*}^{2}\right] \arctan \left(\frac{q_{*}}{2}+\frac{k_{*}}{2}\right)-32 q_{*}^{3}\left(k_{*}^{2}+1\right)^{3} \arctan \left(k_{*}\right) \\
& \left.-32 k_{*}^{3}\left(q_{*}^{2}+1\right)^{3} \arctan \left(q_{*}\right)\right\}\left[24 k_{*}^{2} q_{*}^{2}\left(k_{*}^{2}+1\right)^{3}\left(q_{*}^{2}+1\right)^{3}\right]^{-1}+12 k_{*} q_{*}\left\{\left(q_{*}^{2}-\frac{1}{3}\right) k_{*}^{6}\right. \\
& +\left(2 q_{*}^{4}+\frac{55}{3} q_{*}^{2}+\frac{5}{3}\right) k_{*}^{4}+\left(q_{*}^{6}+\frac{55}{3} q_{*}^{4}+54 q_{*}^{2}+\frac{34}{3}\right) k_{*}^{2} \\
& \left.-\frac{1}{3}\left(q_{*}^{2}+4\right)\left(q_{*}^{4}-9 q_{*}^{2}+2\right)\right\}\left[24 k_{*}^{2} q_{*}^{2}\left(k_{*}^{2}+1\right)^{2}\left(q_{*}^{2}+1\right)^{2}\left[\left(4+k_{*}^{2}+q_{*}^{2}\right)^{2}-4 k_{*}^{2} q_{*}^{2}\right]\right]^{-1} \text {; } \\
& \nu_{0}^{1 s}\left(k_{*}, q_{*}\right)=4\left[\left(4+k_{*}^{2}+q_{*}^{2}\right)^{2}-4 k_{*}^{2} q_{*}^{2}\right]^{-1}+\left(4 k_{*} q_{*}\right)^{-1} \ln \frac{4+\left(k_{*}+q_{*}\right)^{2}}{4+\left(k_{*}-q_{*}\right)^{2}} ; \\
& \nu_{1}^{1 s}\left(k_{*}, q_{*}\right)=\left(2 k_{*} q_{*}\right)^{-1}\left\{\frac{k_{*}^{2}+q_{*}^{2}}{4 k_{*} q_{*}} \ln \frac{4+\left(k_{*}+q_{*}\right)^{2}}{4+\left(k_{*}-q_{*}\right)^{2}}\right. \\
& \left.+\left[4\left(1+k_{*}^{2} q_{*}^{2}\right)-\left(2+k_{*}^{2}+q_{*}^{2}\right)^{2}\right]\left[\left(4+k_{*}^{2}+q_{*}^{2}\right)^{2}-4 k_{*}^{2} q_{*}^{2}\right]^{-1}\right\} \text {. }
\end{aligned}
$$

Mathematical Modeling and Computing, Vol. 7, No. 1, pp. 125-139 (2020) 


\title{
Розрахунок поперечного перерізу фотоіонізації від'ємних іонів водню у борнівському наближенні
}

\author{
Ваврух М. В. ${ }^{1}$, Дзіковський Д. В. ${ }^{1}$, Стельмах О. М. ${ }^{1}$, Солов'ян В. Б. ${ }^{2}$ \\ 1 Львівсъкий начіональний університет імені Івана Франка, \\ вул. Кирила і Мебоділ, 8, 79005, Лъвів, Україна \\ ${ }^{2}$ Інститут фізики конденсованих систем НАН України, \\ вул. Свениічького, 1, 79011, Львів, Україна
}

\begin{abstract}
Показано, що двоелектронна хвильова функція системи "атом водню + фотоелектрон" у борнівському наближенні забезпечує високу точність розрахунку поперечного перерізу процесу фотоіонізації негативного іона водню. Розраховано часткові поперечні перерізи, що відповідають різним "ns-каналам реакції" у шкалі енергій фотоелектрона і шкалі довжин хвиль. Виконано порівняння з результатами інших авторів, одержаних іншими методами.
\end{abstract}

Ключові слова: двоелектронна хвильова функиія, борнівсъке наближення, негативний іон водню, поперечний переріз фотоіонізацї̈. 